\title{
Nanowires and Nanoribbons Formed by Methylphosphonic Acid
}

\author{
B. S. Archanjo, L. A. S. Carvalho, M. Rassa, D. R. Miquita, F. A. C. de Oliveira, \\ L. G. Cançado, U. Agero, F. Plentz, L. A. Cury, J. C. Gonzalez, R. L. Moreira, \\ R. Paniago, R. Magalhães-Paniago, and B. R. A. Neves* \\ Departamento de Física - Universidade Federal de Minas Gerais, UFMG Ave. Antonio Carlos, 6627, Belo Horizonte, MG, Brazil
}

\begin{abstract}
The production and physical properties of nanowires and nanoribbons formed by methylphosphonic acid (MPA) $-\mathrm{CH}_{3} \mathrm{PO}(\mathrm{OH})_{2}$-were investigated. These structures are formed on an aluminum coated substrate when immersed in an ethanolic solution of MPA for several days. A careful investigation of the growth conditions resulted in a narrow window of solution concentrations and temperatures for the successful development of nanowires and nanoribbons. Several different techniques were employed to characterize these nanostructures: (1) Photoluminescence experiments showed a strong emission at $2.3 \mathrm{eV}$ (green), which is visible to the naked eye; (2) X-ray diffraction experiments indicated a significant cristalinity, in agreement with atomic force microscopy (AFM) and transmission electron microscopy (TEM) morphology images, which show organized nano-scale wires and ribbons, (furthermore, AFM-Phase and TEM images also suggest that nanoribbons are formed by well-aligned nanowires); (3) Conductive-AFM experiments revealed an intermediary conductivity for these structures $\left(10^{-1} / \mathrm{Ohm} \cdot \mathrm{m}\right)$, which is similar to some intrinsic semiconductors and; (4) finally, Infrared, Raman, and X-Ray Photoelectron Spectroscopies produced information about the contents, structure, and composition of both wires and ribbons.
\end{abstract}

Keywords: Methylphosphonic Acid, Nanowires, Nanoribbons.

\section{INTRODUCTION}

One of the most motivating features of the ever expanding fields of nanoscience and nanotechnology is the possibility of discovery of novel materials which are only found, and therefore studied, at the nano-scale. Carbon fullerenes and nanotubes are probably the best examples of nanoexclusive materials which began to be explored only when the experimental tools progressed and enabled the access to the nano-scale. ${ }^{1,2}$ The importance of these two structures to current nanoscience and nanotechnology can be measured by the thousands of scientific papers annually published concerning them. Due to the development of experimental tools in the last two decades, further hundreds of new nano-objects of different shapes and compositions have also been created and/or discovered. ${ }^{3}$ The accuracy of modern characterization methods, like some microscopy and spectroscopy techniques, which are able to resolve and detect specimens down to the atomic and molecular scale, created the impression that any new material at the nano-scale could be precisely characterized. ${ }^{3}$ However, even though it has always been possible to see

\footnotetext{
*Author to whom correspondence should be addressed.
}

(through microscopy) and sense (through spectroscopy) such nano-scale materials, their structure determination by X-ray diffraction and/or Nuclear Magnetic Resonance still requires micro-scale quantities $\left(10^{-5} \mathrm{~g}\right.$ to $10^{-4} \mathrm{~g}$, for single-crystals and $\sim 10^{-3} \mathrm{~g}$, for multi-component polycrystalline materials). ${ }^{3}$ So far, this mass constraint has never appeared to be a problem, as nano-scale objects have been routinely produced in such quantities, enabling structural resolution no matter how complex their structure may be. ${ }^{3}$ Nevertheless, a hypothetical complex-structure multicomponent material which is only produced in nano-scale quantities might prove to be very hard, if not impossible, to have its structure fully determined by the methods above. In such a case, a thorough characterization of its accessible physical properties (morphological, optical, and electrical) in addition to structural and compositional information, gathered from several spectroscopy techniques, might be acceptable as a first step on the investigation of a novel material.

The study of phosphonic acid-related materials is multifold: it ranges from the investigations of warfare agents ${ }^{4,5}$ and protective self-assembled films ${ }^{6-8}$ up to catalysts formed by metal-phosphonate compounds. ${ }^{9-13}$ Even when 
focusing on nanomaterials, a widespread number of investigations can be found. ${ }^{14-19}$ A good example of such studies is the work of Maeda and colleagues, who have synthesized a novel material, aluminum methylphosphonate-AlMePO_via a hydrothermal route using bohemite and methylphosphonic acid (MPA) as precursors. ${ }^{10,11}$ The precise structural determination of two polymorphs of AlMePO and the investigation of some of their physical-chemical properties were fully accomplished in their subsequent works. ${ }^{10-13}$ Although AlMePO single-crystals are not nanometric (their dimensions range between hundreds of micrometers and several millimeters $),{ }^{10-13}$ the presence of one-dimensional organic lined channels with nanometric diameter envision several nano-related applications, such as catalysis and selective transport. ${ }^{10-13}$ In another interesting work, Lewington and co-workers investigated the surface reactivity of aluminum when covered by self-assembled monolayers of phosphonic acids with different alkyl chains. ${ }^{6}$ The samples were prepared by immersion of an Al-coated substrate in an ethanolic solution of these acids and it was found that phosphonic acids with long alkyl chains provided the best stability for the aluminum surface when it was previously immersed for 15 minutes in a MPA solution. ${ }^{6}$ Extending Lewington's investigation, in the present work, Al-coated substrates were immersed in ethanolic solutions of MPA for much longer times (up to 100 days). It was found that, after a few days of immersion (typically, 2 to 3 days), nanowires and nanoribbons develop at the Al surface. Following this initial result, a careful investigation about the conditions (solution concentration, immersion time, temperature, reagents) for the production of such nanostructures was carried out. Concomitantly, their physical properties (morphological, electrical, and optical) were assessed using optical microscopy (OM), atomic force microscopy (AFM), scanning electron microscopy (SEM), transmission electron microscopy (TEM), conductive-AFM, and photoluminescence spectroscopy (PL). As a consequence of the observation of interesting physical properties (strong luminescence and intermediate conductivity), the composition and structure of both nanowires and nanoribbons were further investigated by X-ray diffraction, Infrared (IR), Raman, and X-ray Photoelectron (XPS) spectroscopies. The results indicate a complex (and not previously reported) MPArelated compound forming nanowires and nanoribbons, with indications that $\mathrm{Al}$ atoms and ethyl radicals are also incorporated into its structure. Furthermore, there are strong morphological evidences that ribbons are formed by well-aligned wires. Both nanowires and nanoribbons are stable to heat treatments up to $250{ }^{\circ} \mathrm{C}$, but their luminescence suffers an aging effect after several months at ambient conditions. In summary, this work reports on several interesting physical properties of nanowires and nanoribbons formed by a MPA-compound, along with some indications of its compositional and structural aspects.

\section{EXPERIMENTAL DETAILS}

Methylphosphonic acid- $\mathrm{CH}_{3} \mathrm{PO}(\mathrm{OH})_{2}$-was used as purchased from Alfa Aesar (98\% purity) and dissolved in absolute ethanol (electronic grade-purity 99.8\%) in order to prepare MPA solutions with concentrations ranging from $1 \mathrm{mM}$ up to $25 \mathrm{mM}$. Aluminum, titanium, copper, and nickel (all of them purchased from Kurt J. Lesker Company with $99.99 \%$ purity) films with $\sim 100$ nm thickness were evaporated onto different substrates (silicon, glass, and mica) using a Balzers BAE250 Coating System or a home-made sputtering equipment (a RF generator RFX-600 coupled whit a ATX-600 both from Advanced Energy, a Sputter Head Model 720, and a Depositing Controller Model 860 from Telemark). This system is evacuated, via a $\mathrm{N}_{2}$ trap, by a diffusion oil pump coupled with a mechanical pump and the base pressure can reach $5 \mu$ torr. The argon flux is controlled by a mass flow controller. The working pressure is about 2 mtorr and the applied power is about $200 \mathrm{~W}$. The samples were produced by immersion of Al-coated substrates in MPA ethanolic solution inside a closed recipient. The immersion time varied from $1 \mathrm{~h}$ up to 100 days and the solution temperature varied from $-5{ }^{\circ} \mathrm{C}$ up to $55^{\circ} \mathrm{C}$. After preparation, the substrates were removed from solution, rising for $10 \mathrm{~s}$ in pure ethanol with a vertical movement and dried with pure nitrogen $(99 \%$ purity) at an approximated angle of 45 degrees. The AFM morphological investigation was carried out using a Veeco Instruments Nanoscope IV MultiMode SPM operating in intermittent contact mode under a relative humidity of $50 \%$ at a laboratory temperature of $22{ }^{\circ} \mathrm{C}$ (scan rate: $1.3 \mathrm{~Hz}$, engaged amplitude: $70 \%$ of free oscillation amplitude and using conventional silicon probes-resonant frequency: $300 \mathrm{KHz}$ and spring constant: $40 \mathrm{~N} / \mathrm{m}$ ). Conductive-AFM experiments were performed with a NT-MDT Solver Pro SPM operating in contact mode using Pt-coated silicon probes (cantilever spring constant $=0.1 \mathrm{~N} / \mathrm{m}$ ) under a relative humidity of $50 \%$ at a laboratory temperature of $22{ }^{\circ} \mathrm{C}$. During conductive-AFM imaging, a constant bias is applied between sample and probe and the resulting current is recorded for each position (image pixel), creating a current (conductivity) map of the sample. I(V) characteristic curves can also be obtained at any sample region by positioning the probe at such location and recording the tip-sample current as the bias is swept. SEM images were acquired with a Jeol 840A microscope operating at $25 \mathrm{kV}$ and a current of $15 \mathrm{pA}$. TEM images were obtained with a Jeol HRTEM-JEM 3010 URP electron microscope operating at $300 \mathrm{kV}$ with a point resolution of $0.17 \mathrm{~nm}$. $\mathrm{X}$-ray diffraction data were acquired with a $200 \mu \mathrm{m} \times$ $2 \mathrm{~mm}$ collimated beam of wavelength $\lambda=1.9088 \AA$. The scattering was collected by a scintillation detector with a Hubber four-circle diffractometer at the XRD2 beamline of the National Synchrotron Light Laboratory (LNLS) in Campinas, Brazil. Photoluminescence spectra were obtained at room temperature using a Coherent $\mathrm{Ar}$ 
laser $(\lambda=488 \mathrm{~nm})$ and a SPEX-CD2A monochromator with a photomultiplier detector and controller. A filter cutting light wavelengths below $515 \mathrm{~nm}$ was also employed at the entrance of the spectrometer to protect the photodetector. Infrared spectroscopy was accomplished with a Nicolet Nexus470 FTIR spectrometer with a HgCdTe detector, combined with a Centaurus microscope with a $10 \mathrm{X}$ objective, a $\mathrm{SiC}$ source, and a $\mathrm{KBr}$ beam splitter. Nanowires and nanoribbons were scraped from their substrates and IR spectra were collected focusing the beam on a single agglomerate of scraped material and collecting the reflected light. All measurements were corrected for background removal. Backscattering micro Raman spectra were taken at room temperature using a DILOR XY triple monochromator. The laser energy, spot area, and power density on the sample were $2.41 \mathrm{eV}, 10^{-8} \mathrm{~cm}^{2}$, and $3 \times 10^{5} \mathrm{~W} / \mathrm{cm}^{2}$, respectively. Finally, XPS spectra were obtained with a VG-Escalab 220i-XL system with nonmonochromatized Al-K $\alpha$ radiation $(E=1486.6 \mathrm{eV})$. The hemispherical analyzer was operated with pass energy of $20 \mathrm{eV}$, corresponding to an energy resolution of $0.8 \mathrm{eV}$. All spectra were taken at normal emission. For evaluation of the XPS-data, a Shirley-type background correction in the Eclipse software (VG-Scientific) was used. The sensitivity factors used for quantitative analysis were 0.63 (Al 2p), 1.18 (P 2s), and 1.19 (P 2p), respectively.

\section{RESULTS AND DISCUSSION}

Figure 1 shows representative AFM images of nanowires and nanoribbons formed in different samples after 7 days immersion in a $9 \mathrm{mM}$ MPA-ethanol solution at room temperature. Casually, all these images were acquired with samples prepared on $\mathrm{Si}$ substrates. Nevertheless, the results are similar regardless the employed substrate. In Figure 1(a), tens of nanowires, with diameters ranging from $10 \mathrm{~nm}$ up to $50 \mathrm{~nm}$ and lengths from hundreds of nanometers up to few microns, can be seen. Figure 1(b) portrays a sample which has nanoribbons only. These nanoribbons present a rectangular shape, with 10 to $20 \mathrm{~nm}$ thickness, 100 to $200 \mathrm{~nm}$ width and a few microns in length. Figure 1(c) shows an image of a sample which presents both nanowires and nanoribbons (beneath the wires in Fig. 1(c)). In summary, there are samples with nanowires only Figure 1(a), with nanoribbons only Figure 1(b) and with both wires and ribbons Figure 1(c). Higher magnification AFM images in Figures 1(d) and (e) show details of nanowires and nanoribbons, respectively.
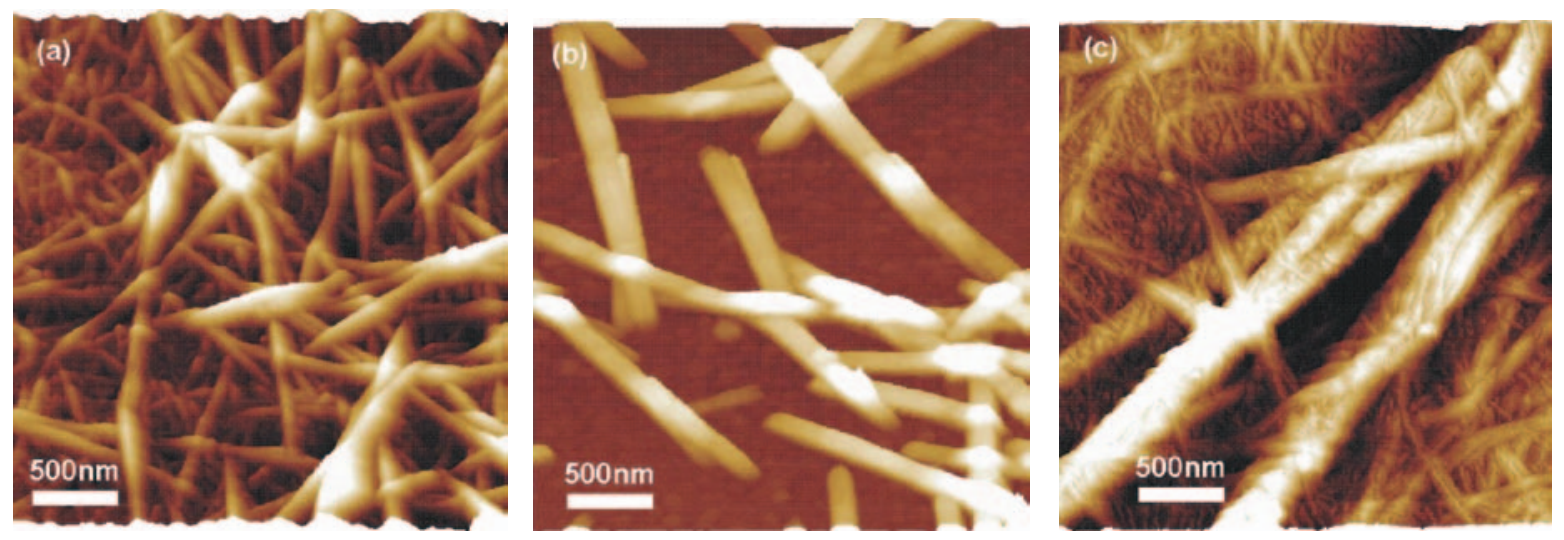

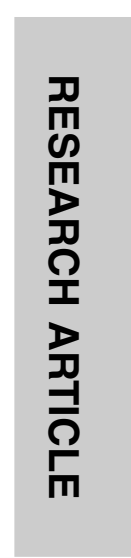
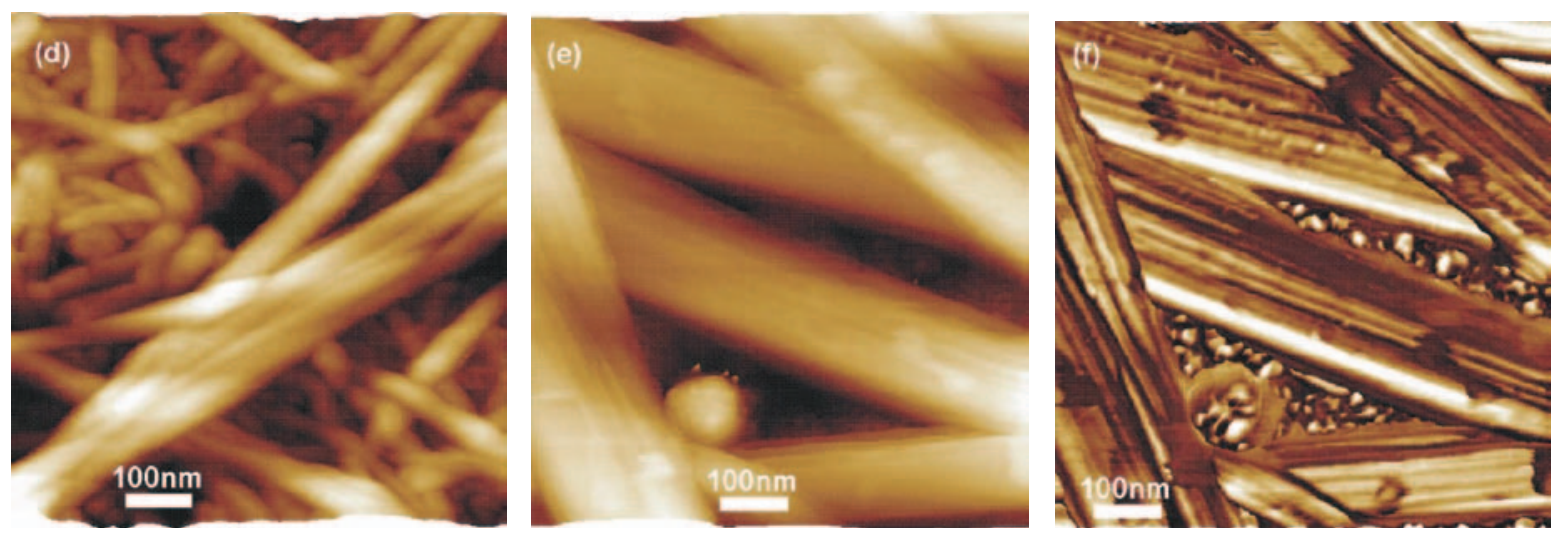

Fig. 1. (Color online) AFM images of nanowires and nanoribbons produced with a $9 \mathrm{mM}$ MPA-ethanol solution and 7 days of immersion time using silicon substrates. Figures (a) through (e) are 3D topographical AFM images, while figure (f) is a phase contrast AFM image. A scale bar at the bottom of each image indicates its lateral dimensions. The z-scale (color coded-from black to white) in figures (a), (b), and (e) is $100 \mathrm{~nm}$; and it is $200 \mathrm{~nm}$, $50 \mathrm{~nm}$, and $30^{\circ}$ in figures (c), (d), and (f), respectively. 


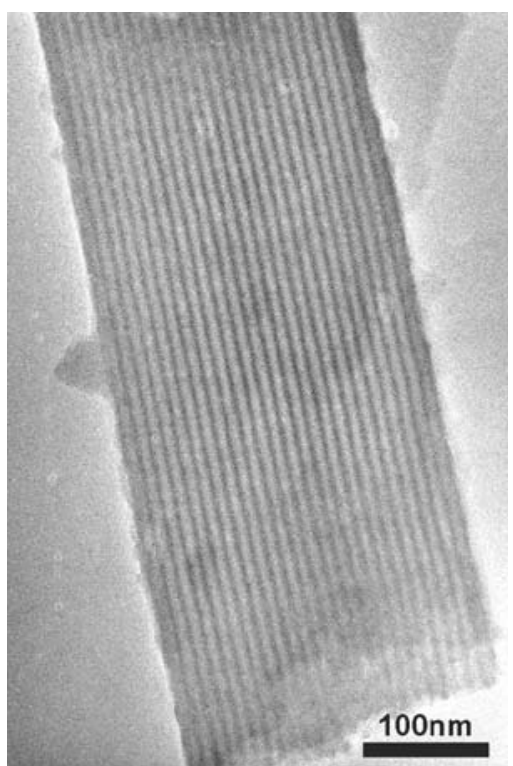

Fig. 2. TEM micrograph of a free-standing nanoribbon. The scale bar at the bottom right of the image indicates its dimensions.

Indeed, looking carefully at the phase contrast AFM image in Figure 1(f), acquired simultaneously with Figure 1(e), there is an indication that nanoribbons may actually be formed by aligned filaments. Although phase contrast AFM images are not very sensitive to height variations, they are very sensitive to topographic features, like borders, and to compositional variation, enabling, thus, differentiation of apparently smooth samples. ${ }^{20}$ Such structural hypothesis is verified in the TEM image of a single nanoribbon in Figure 2, which clearly shows aligned filaments forming this nanoribbon. The average filament diameter in Figures 1(f) and 2 is $\sim 10 \mathrm{~nm}$, which is similar to the smallest nanowires in Figures 1(a), (c), and (d). Therefore, it is plausible that nanoribbons might be formed by aligned nanowires and, then, the wire structure could be conceived as the primary building block of this system.

Another interesting morphological difference between nanowires and nanoribbons is that nanowires are always evenly distributed on the sample surface, as indicated by Figures 1(a) and (c), whereas nanoribbons often form large (sub-milimetric) interconnected aggregates, as shown in Figure 3. Figures 3(a) and (b) show OM images of the sample with the largest concentration of nanoribbons produced in this work (30 days immersion on a $8 \mathrm{mM}$ solution). It is clear in these images the interconnection between the star-shaped agglomerates (dark regions) on top of glass substrate (light regions). A closer look at an agglomerate reveals a large amount of intertwined nanoribbons, as shown by the SEM image in Figure 3(c).

Before analyzing the physical properties of nanowires and nanoribbons any further, it is necessary to discuss the formation process of these structures. As already said above, several experimental parameters were varied in order to investigate their influence on the formation of
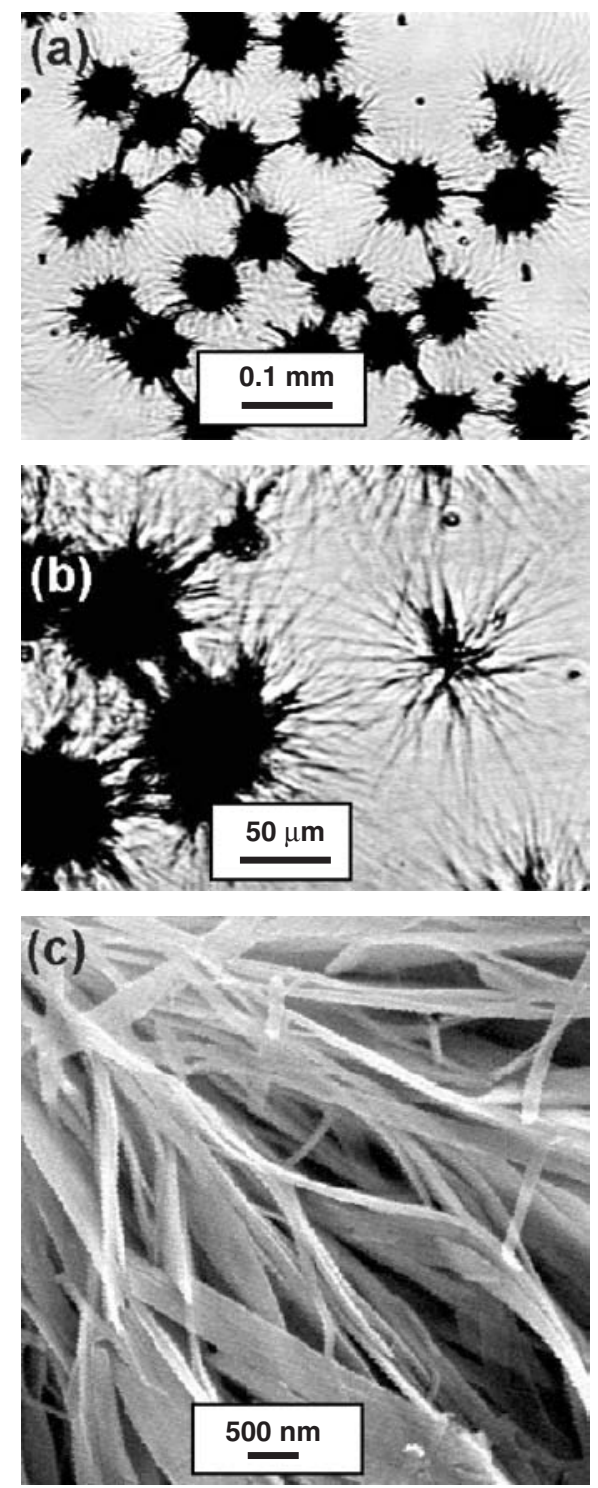

Fig. 3. ( $a$ and b) OM images and (c) SEM image of nanoribbons formed after immersion for 30 days of the $\mathrm{Al}$ substrate in a $8 \mathrm{mM}$ MPAethanol solution. A scale bar at the bottom of each image indicates its dimensions.

these nanostructures. Initially, solution concentration and immersion time were examined. Several MPA-ethanol solutions were prepared with concentrations ranging from $1 \mathrm{mM}$ up to $25 \mathrm{mM}$ and the immersion time of the Al-coated substrate varied from $1 \mathrm{~h}$ up to 100 days. Unexpectedly, only a short range of solution concentrations (from $7 \mathrm{mM}$ up to $10 \mathrm{mM}$ ) was found to produce either nanowires and/or nanoribbons. Solutions with concentration below $7 \mathrm{mM}$ do not produce any of these nanostructures and, actually, no apparent morphological modification of the $\mathrm{Al}$ film is observed within the time span of the experiments (up to 100 days). Solutions with concentration above $12 \mathrm{mM}$ do not produce nanowires or nanoribbons either, but morphological modifications of the $\mathrm{Al}$ film are observed. Its fast consumption along time 
is clear, indicating a chemical reaction with the solution. However, no nanostructure (wire or ribbon) was ever observed on any substrate after it was removed from solution. It was conjectured that any reaction products could be afloat inside solution and not attached to the substrate. Several attempts to collect any products by strong centrifugation of the concentrated solutions were made without any success, i.e., no wire or ribbon was ever observed. Possibly, at higher MPA concentrations (and media acidity) soluble complex species might be formed. Such hypothesis can be substantiated by the low $\mathrm{pK}_{a}$ of MPA and its high stability constant for binding of two molecules on a single $\mathrm{Al}$ atom. ${ }^{19,21}$ Nevertheless, whichever route MPA molecules take at high concentration, for the present work, the important result is the net effect of no nanowire or nanoribbon observation and, thus, any further discussion regarding high concentration solution falls beyond the scope of the present work.

The effect of immersion time, for solutions between $7 \mathrm{mM}$ and $10 \mathrm{mM}$, is orthodox: ${ }^{21}$ there is a minimum time ( $\sim 2$ days) for the initial formation of small wires and ribbons; as time passes (between 3 and 30 days), the size and amount of wires and/or ribbons increases; but longer immersion times (more than 30 days) do not yield larger amounts of nanostructures, probably indicating the consumption of a reagent (MPA, most likely) after such immersion times. These results indicate a very slow formation (reaction) rate for both nanowires and nanoribbons, and efforts to increase it were also carried out by investigating the effects of temperature on their formation. As expected, cooling the solution temperature down to $-5{ }^{\circ} \mathrm{C}$ drastically decreased the formation of nanostructures, precluding the formation (and observation) of any ribbons or wires even after weeks of immersion at such temperature. ${ }^{21}$ However, it was also observed that increasing the temperature does not increase the formation rate of wires and ribbons. Actually, the room temperature $\left(20^{\circ} \mathrm{C}\right.$ to $\left.25^{\circ} \mathrm{C}\right)$ was found to present the highest formation rate, which, then, decreases steadily as temperature is raised up to $55{ }^{\circ} \mathrm{C}$, the highest investigated in this work. Such unexpected result may indicate the presence of two competing reactions: wire and/or ribbon formation versus esterification of MPA. If such esterification is more exothermic than nanostructure formation, than it might prevails as temperature is increased, precluding wire/ribbon formation. Again, for the present work, any detailed discussion of any competing reaction is out of range and is left for a subsequent study.

Additionally, any eventual effects of substrate nature on the formation of wires and ribbons were also considered. Consequently, a batch of samples was prepared using different substrates-mica, glass, and silicon, and again, no effect of substrate was observed, i.e., nanowires and nanoribbons were formed regardless the substrate. The importance of each reagent-MPA, ethanol, and Alwas also checked in a series of tests where each reagent was consecutively substituted by a similar compound. In such a way, MPA was substituted by propylphosphonic acid, phenylphosphonic acid, or benzylphosphonic acid; ethanol was substituted by methanol, hydrated ethanol, isopropanol, tetrahydrofuran, or water; and $\mathrm{Al}$ was substituted by $\mathrm{Ti}, \mathrm{Ni}$, or $\mathrm{Cu}$. The only reagent combination which produced nanowires and nanoribbons was MPA, ethanol, and Al. It is interesting to observe that even the smallest amount of water in the ethanol totally precludes the formation of these nanostructures, in a clear contrast with the formation of AlMePO, which always proceeds in aqueous solution. ${ }^{10-13}$ A possible explanation for this observation is that water could lead to formation of oxohydroxoaluminum compounds at the sample surface, increasing proton activity. Thus, such compound would preferentially react with MPA yielding different species rather than wires and ribbons. Finally, it must be stressed that despite innumerous attempts, it was not possible in the present work to discriminate the experimental conditions for selective formation of nanoribbons or nanowires. Apparently, fresher solutions have a tendency to produce nanowires more often than nanoribbons and aged, or recently used, solutions produce nanoribbons more often than nanowires. Such behavior may indicate that the formation of nanoribbons might occur at a slower rate when compared to the formation of nanowires, which agrees with the hypothesis that nanoribbons are actually formed by aligned nanowires. Finally, the thermal stability of nanowires and nanoribbons was tested by annealing samples in a conventional oven up to $250{ }^{\circ} \mathrm{C}$ for $1 \mathrm{~h}$. Each sample was characterized (morphology and spectroscopy-AFM, PL, IR, and XPS) before and after the annealing procedure and no morphological or spectroscopic modification was ever observed, indicating a good stability of such nanostructures upon thermal treatments up to $250{ }^{\circ} \mathrm{C}$.

The immediate consequence of such narrow window of optimal conditions for the formation of wires and ribbons is that, within the time frame of several months, only a very small amount of material could be produced. Such mass scarceness posed an unsurpassable barrier during attempts to determine the actual atomic structure of nanowires and nanoribbons, as described in the following paragraphs. Nevertheless, a great amount of information about these nanostructures was assembled using conventional techniques. Two examples of such information are presented in Figures 4 and 5, which portray some optical and electrical properties of nanowires and nanoribbons.

Figure 4 shows photoluminescence spectra obtained with a sample with nanowires only (blue), a sample with nanoribbons only (black) and MPA (red). All structures show a pronounced and broad peak centered at $553 \mathrm{~nm}-$ $2.24 \mathrm{eV}$ (nanowires and MPA) or $530 \mathrm{~nm}-2.34 \mathrm{eV}$ (nanoribbons). The asymmetric shape of this peak is due to the presence of the $515 \mathrm{~nm}$ filter. No other PL peak was observed up to $850 \mathrm{~nm}$ for any structure. The resemblance 


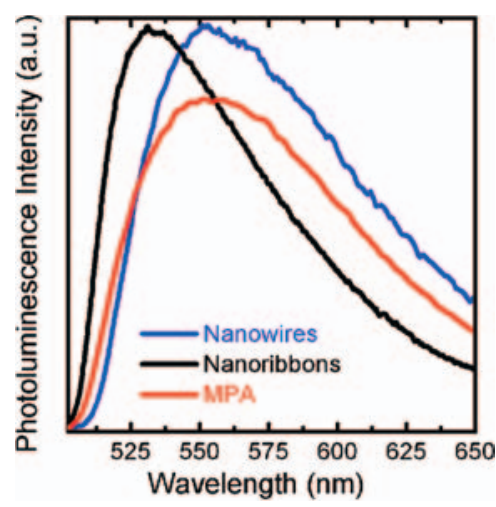

Fig. 4. (Color online) Photoluminescence spectra of nanowires (blue), nanoribbons (black), and MPA (red).

of the PL spectra of MPA and nanowires indicate that whatever the nanowire composition and structure might be, its radiative center is exactly the same of MPA. In other words, the presence of different MPA neighbors in the nanowire structure does not affect the energy levels, or molecular orbitals, responsible for the radiative recombination in the MPA molecule. ${ }^{22}$ Even though the
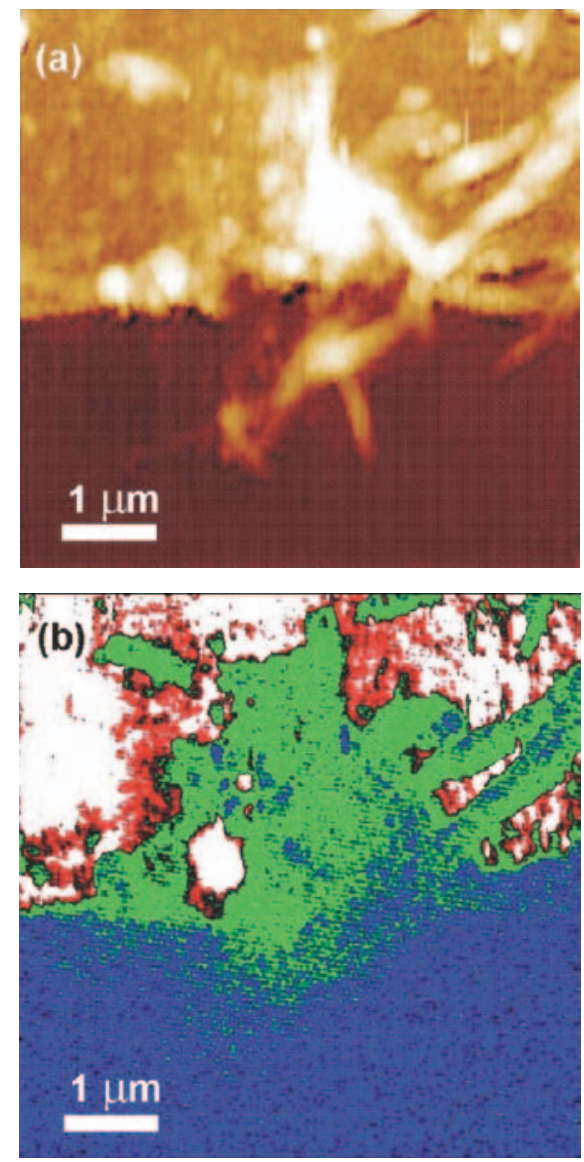

Fig. 5. (Color online) Conductive-AFM characterization of nanowires. Figure (a) shows an AFM topographic image of the sample and Figure (b) shows a current map (blue-no current; green-low current; and redwhite-high current) of the same region. A scale bar at the bottom left of each image indicates its dimensions. radiative center of MPA is not exactly known, it can be conjectured that it has the same nature of some phosphonic and phosphoric acid derivatives, which are known chromophores. ${ }^{23,24}$ In such substances, the lumophore center is normally associated to the P atom..$^{23,24}$ The PL spectrum of nanoribbons shows a small shift to higher energies, suggesting a possible additional confinement of the MPA radiative center within the nanoribbon structure. ${ }^{22,25} \mathrm{Nev}-$ ertheless, the most striking optical property of nanoribbons is its recombination efficiency (quantum yield): when focusing the laser on one agglomerate, like those in Figure 3, its luminescence is so intense that it can be easily seen with the naked eye (wearing special goggles which eliminate the exciting laser light). However, such efficiency decreases with time (in a timescale of months), indicating an aging effect of these nanostructures.

Figure 5 presents conductive-AFM analysis of a special sample with nanoribbons. A photolithographic process was carried out to define thick Al tracks (yellowish or light region in Fig. 5(a)) on the glass substrate (brownish or dark region in Fig. 5(a)). Nanoribbons protruding from the remaining Al track onto the bare glass substrate were formed after 7 days immersion in a $8 \mathrm{mM}$ solution as shown in Figure 5(a). The current map of this same region, shown in Figure 5(b), shows no electrical current flowing into the glass substrate (blue region), a small current $(\sim 20 \mathrm{pA})$ flowing into the nanoribbons (green region) and a larger current $(\sim 60 \mathrm{pA})$ flowing into the $\mathrm{Al}$ track (whitered region). Such image indicates that nanoribbons have an intermediary conductivity, which is larger than that of an insulator (glass), but smaller than that of a metal (Al). A more quantitative analysis can be performed by acquiring $\mathrm{I}(\mathrm{V})$ curves at different regions of the sample and measuring current values at each location for a given bias. Making a very simple model for the current flow through a cone trunk, where one base has the diameter of the Ptcovered probe and the other cone base has the diameter of the nanoribbon width, and accounting for the series resistance of the $\mathrm{Al}$ film below the nanoribbon, its conductivity is estimated to be $\sim 10^{-1} / \mathrm{Ohm} \cdot \mathrm{m}$, which is similar to some intrinsic semiconductors. ${ }^{25,26}$

Following the discovery of interesting optical and electrical properties (Figs. 4 and 5), it became clear that a thorough investigation on the structure and composition of nanoribbons and nanowires was needed. The most obvious step in such direction was a survey of the crystalline structure of wires and ribbons by X-ray diffraction. Therefore, a synchrotron radiation X-ray source was employed, enabling the acquisition of several diffractograms from nanowires, nanoribbons, and the MPA precursor. Figure 6 shows some typical results obtained from samples with nanowires (blue), nanoribbons (black), and MPA (red). Several sharp diffraction peaks can be seen in the nanoribbon and nanowire scans and tens of peaks are found in the MPA scan. The nanoribbons diffractogram was 


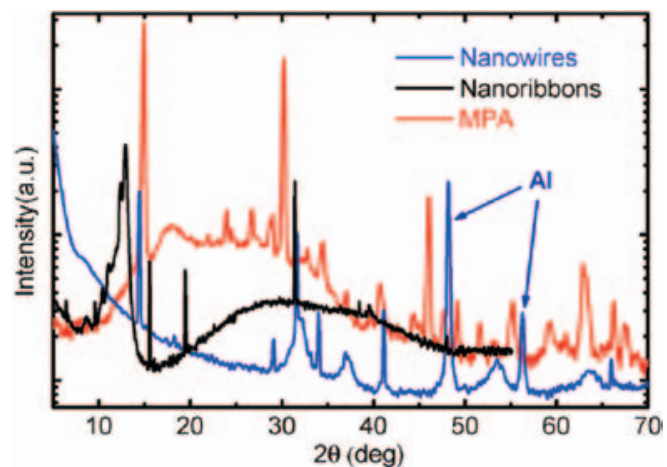

Fig. 6. (Color online) X-ray diffractograms from nanowires (blue), nanoribbons (black), and MPA (red). The intensity is plotted in a logarithmic scale.

measured from a sample prepared on a glass substrate (see Fig. 3), and, therefore, the broad halo between $17^{\circ}$ and $46^{\circ}$ comes from scattering in the amorphous glass substrate. ${ }^{27,28}$ The diffractograms of both nanowires and MPA were acquired with the material on a $\mathrm{Si}(100)$ substrate and, thus, do not suffer such interference. However, the broad halo seen between $8^{\circ}$ and $45^{\circ}$ in the MPA diffractogram comes from scattering in the Kapton ${ }^{\circledR}$ film employed to prevent hydration of the MPA material. It is interesting to note that, although MPA is a well investigated material, mainly due to its warfare applications, ${ }^{4,5}$ when trying to confirm its crystal structure, no entries on the literature could be found.

In the nanowires diffractogram, there are two peaks (indicated by arrows in Fig. 6) which originated from diffraction on the remaining metallic Al. In such sample, a thick $\mathrm{Al}$ film was employed (in an effort to produce more material), resulting in a good amount of non-reacted metallic $\mathrm{Al}$ beneath the nanowires after sample preparation. The first interesting result observed in Figure 6 is the presence of sharp peaks in the diffractograms of both nanowires and nanoribbons, which clearly indicate some degree of cristalinity of these structures. ${ }^{27,28}$ In other words, nanowires and nanoribbons are not (totally) amorphous, whatever their composition and structure might be. However, the reduced number of peaks in each diffractogram precludes completely the determination of its pertinent crystal structure, especially when taking into consideration that the samples are polycrystalline with a preferential orientation (texture), as can be seen in Figures 1 and 3.. ${ }^{27,28}$ Moreover, even though there are some common peaks among the nanowire, nanoribbon, and MPA diffractograms, it is evident from Figure 6 that wires and ribbons present completely different crystal structures from MPA. It is also interesting to note that most peaks from nanoribbons are located at small angles, indicating large interplanar distances (as large as $48.7 \AA$ ), whereas nanowire peaks are at larger angles, denoting smaller interplanar distances. In other words, the nanoribbon unitary cell is probably larger than the nanowire one. ${ }^{27,28} \mathrm{~A}$ careful inspection of the nanowire diffractogram in Figure 6 reveals two distinct peak series: a sharp peak and a broad peak series. Measuring the average peak width on each series and applying Scherrer equation, it is possible to estimate the average size of the diffracting specimen. ${ }^{27,28}$ Within such analysis, the sharp peak series comes from diffracting objects more than a hundred nanometers large and the broad peak series arises from objects which are $\sim 10 \mathrm{~nm}$ in size. Such length is similar to the diameter of the smaller nanowires shown in Figures 1 and 2, suggesting the possible origin of this series.

Even though some basic information about the structure of nanowires and nanoribbons can be extracted from the diffractograms in Figure 6, it is not possible to extract the actual crystal structure of either. Normally, hundreds, or even thousands, of diffraction peaks are needed for a successful peak indexing and structure determination by standard procedures, like the Rietveld method. ${ }^{27,28}$ This method relies on an isotropic distribution of crystallites in a polycrystalline sample, which, experimentally, requires $\sim 10^{-3} \mathrm{~g}$ of material. ${ }^{27,28}$ Such required amount is orders of magnitude larger than the total amount of nanostructures produced throughout this work. Therefore, nanowire and nanoribbon structural determination by X-ray diffraction was hindered by their mass scarceness. Nevertheless, additional compositional and structural information was obtained with different spectroscopic techniques, as shown in Figures 7, 8, and 9.

Figure 7 summarizes the XPS investigation of nanoribbons and nanowires. The same samples investigated by X-ray diffraction were used in the XPS study. Nanoribbons were grown on a glass substrate and no residual metallic $\mathrm{Al}$ was found on the sample either by microscopy analysis or X-ray diffraction, indicating that the entire $\mathrm{Al}$ film was consumed during the formation of this sample. Again, no metallic Al could be seen in this XPS study (lower black spectrum in Fig. 7). Nanowires were observed upon a residual metallic $\mathrm{Al}$ film on $\mathrm{Si}$ substrate as evidenced

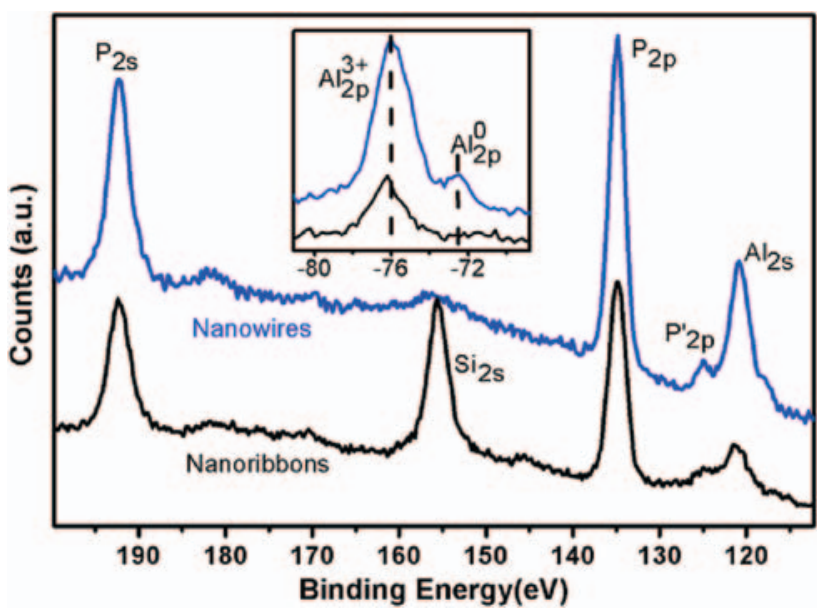

Fig. 7. (Color online) XPS spectra of nanowires (blue) and nanoribbons (black). The inset evidences Al-related peaks on both structures. 


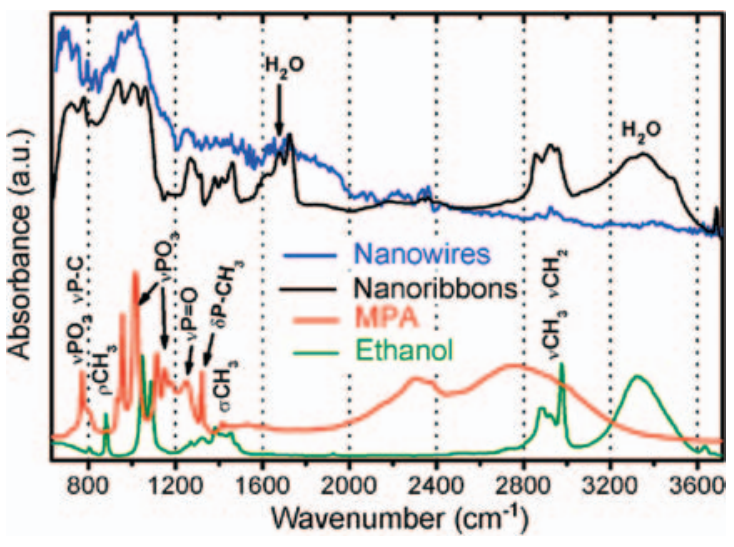

Fig. 8. (Color online) Infrared absorption spectra of nanowires (blue), nanoribbons (black), MPA (red), and ethanol (green). Several peaks are indexed in the figure.

by strong $\mathrm{Al}$ peaks in their XPS spectrum in Figure 7. Due to surface charging of the sample during XPS measurements, spectra were corrected using the P-2s peak at $192.5 \mathrm{eV}$ binding energy. ${ }^{30}$ With this correction, spectrum of nanowires gave binding energies of $72.8 \mathrm{eV}$ for the $\mathrm{Al}_{2 \mathrm{p}}^{0}$ energy core level, which agrees with reference $30(72.6 \mathrm{eV})$. All main visible peaks in both spectra of Figure 7 have been indexed. The peaks centered at electron binding energies of $135.1 \mathrm{eV}, 121.1 \mathrm{eV}$, and $76.1 \mathrm{eV}$ on the nanowires sample (upper blue spectrum) are associated to $\mathrm{P}_{2 \mathrm{p}}, \mathrm{Al}_{2 \mathrm{~s}}$, and $\mathrm{Al}_{2 \mathrm{p}}^{3+}$ core levels, respectively. ${ }^{30,31}$ The small peak centered at $125.3 \mathrm{eV}\left(\mathrm{P}_{2 \mathrm{p}}^{\prime}\right)$ is a satellite of the $\mathrm{P}_{2 \mathrm{p}}$ peak. ${ }^{26,29}$ All these four peaks are also present on the nanoribbons spectrum, but the $\mathrm{Al}_{2 \mathrm{p}}^{3+}$ energy core level is slightly dislocated to $76.3 \mathrm{eV}$ and there is an additional peak centered at $155.7 \mathrm{eV}$ which is associated to $\mathrm{Si}_{2 \mathrm{~s}}$ core level from $\mathrm{SiO}_{x}$ on glass substrate.

The analysis of Figure 7 indicates that $\mathrm{Al}$ atoms on both nanoribbons and nanowires are on oxidation state +3 , which is similar to the case of MPA adsorbed onto $\mathrm{Al}(111) .^{30}$ Probably, in both nanostructures, the $\mathrm{Al}$ atom

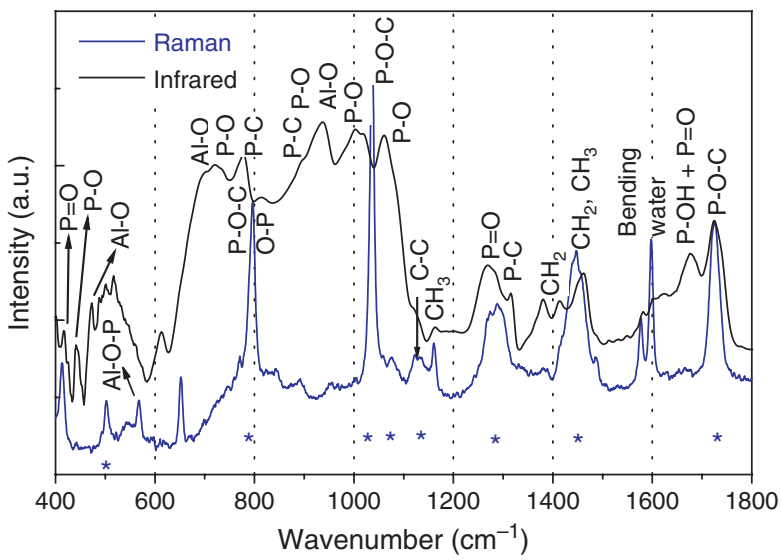

Fig. 9. (Color online) Detailed Raman (blue) and infrared absorption (black) spectra of nanoribbons with the majority of peaks already identified. is linked to MPA by an $\mathrm{Al}-\mathrm{O}$ bond. Although Davies and Newton ${ }^{30}$ do not mention the $\mathrm{P}_{2 \mathrm{p}}$ energy core level, the work by Viinikanoja and co-workers ${ }^{31}$ and references therein, reported the electron binding energy of the $\mathrm{P}_{2 \mathrm{p}}$ core level varying between $132.2 \mathrm{eV}$ and $133.4 \mathrm{eV}$ when the phosphonic acid forms a complex with metallic species. Since this peak is found at $135.1 \mathrm{eV}$ on both nanostrutures shown on Figure 7, the Al-MPA interaction within the nanoribbons has probably a different nature and might not be a simple adsorption. Applying Gaussian fitting procedures to both $\mathrm{P}_{2 \mathrm{p}}$ and $\mathrm{Al}_{2 \mathrm{p}}$ peaks in Figure 7 and employing the appropriate atomic sensitivity factors, ${ }^{32}$ $\mathrm{P}: \mathrm{Al}$ ratios are found to be 3.2 on nanoribbons and 1.4 on nanowires. This large difference on P:Al ratios supports the idea that nanowires might be an initial step on nanoribbons formation.

The tiny amount of nanowires on any given sample (typically, 100 to 1000 times less material than a nanoribbon sample) affected the investigation of nanowires by optical spectroscopy techniques, like infrared absorption and Raman scattering: it was not possible to acquire acceptable signal-to-noise ratio Raman spectra of nanowires and even their infrared absorption spectra present a much lower quality. Nevertheless, the analysis of the attainable infrared and Raman spectra led to interesting insights on the structure and composition of both nanowires and nanoribbons. Figures 8 and 9 present absorption infrared spectra of nanowires, nanoribbons, MPA, and ethanol; and detailed infrared and Raman spectra of nanoribbons, respectively. Comparing, initially, both nanoribbon and nanowire absorbance spectra in Figure 8 (upper plots), it is easy to verify the presence of absorbed water and ethanol in the nanoribbons sample, as indicated by the bands around $1610 \mathrm{~cm}^{-1}$ and $3350 \mathrm{~cm}^{-1}$ (characteristic of water); and the sharp peak at $3690 \mathrm{~cm}^{-1}$ together with the bands around $2800 \mathrm{~cm}^{-1}$ and $3000 \mathrm{~cm}^{-1}$ (characteristic of ethanol-see lower green plot). ${ }^{33}$ The main resemblance between nanoribbon and nanowire spectra is the broad band between $630 \mathrm{~cm}^{-1}$ and $1100 \mathrm{~cm}^{-1}$, which may originate from several different vibration modes $\nu \mathrm{PO}_{2}, \nu \mathrm{PO}_{3}$, $\nu \mathrm{P}-\mathrm{C}, \rho \mathrm{CH}_{3}, \nu \mathrm{C}-\mathrm{C}$, and $\nu \mathrm{C}-\mathrm{O} .{ }^{32-38} \mathrm{~A}$ detailed view of this band, with respective vibration association, is shown in Figure 9. ${ }^{34-39}$ A comparison between these spectra with those in Ref. [34], which investigated MPA adsorption on alumina, suggests that both nanostructures are not only MPA adsorbed onto alumina, as their MPA-related bands are much more active than those in Ref. [34]. ${ }^{29}$ The bands around $1250 \mathrm{~cm}^{-1}$ and $1300 \mathrm{~cm}^{-1}$, present in both Raman and infrared spectra of nanoribbons (see Fig. 9), are associated to $\delta \mathrm{P}-\mathrm{CH}_{3}$ vibration modes whereas the peak at $\sim 800 \mathrm{~cm}^{-1}$ in both nanoribbon and nanowire infrared spectra (Fig. 8) is associated to $\nu \mathrm{P}-\mathrm{C}$ mode. ${ }^{33-38}$ Therefore, the adsorption does not result on $\mathrm{P}-\mathrm{C}$ cleavage as could be suspected from dephosphorilation pathway in metalloenzymes. Another interesting observation is a 
$20 \mathrm{~cm}^{-1}$ shift of the $\nu \mathrm{P}=\mathrm{O}$ band in both nanowires and nanoribbons when compared with the same band of MPA (which is located at $1250 \mathrm{~cm}^{-1}$ ) - see Figures 8 and 9 . Such shift is normally attributed to a substitution of a hydrogen atom in the $\mathrm{P}-\mathrm{O}-\mathrm{H}$ structure. ${ }^{29,33,35}$ In the case of the MPA molecule, the $\mathrm{H}$ atom of the $\mathrm{OH}$ group interacts with the $\mathrm{O}$ atom in the $\mathrm{P}=\mathrm{O}$ group, leading to a decrease of its resonant frequency when the $\mathrm{H}$ atom is removed or substituted. ${ }^{29,33}$ Additionally, some peaks with low frequencies (below $600 \mathrm{~cm}^{-1}$ ) in both Raman and infrared spectra of nanoribbons in Figure 9 suggest the existence of $\mathrm{Al}-\mathrm{O}-\mathrm{P}$ bonds, indicating an incorporation of Al into their structure. ${ }^{33}$ Finally, it is interesting to compare the Raman spectrum of nanoribbons with the Raman spectra of two related compounds, ethyl methyl phosphonate (EMP), and diethyl methyl phosphonate (DEMP), which present one or two ethyl radicals substituting $\mathrm{OH}$ radicals in the MPA molecule, respectively. ${ }^{39}$ All peaks marked by an asterisk in Figure 9 are present in the Raman spectrum of DEMP and most of them are also present in the spectrum of EMP. ${ }^{39}$ Therefore, it seems reasonable to interpret this result as an evidence of the incorporation of ethyl radicals from ethanol into the MPA core during the formation of nanoribbons. More specifically, an ethanol molecule reacts with each hydroxyl group of the MPA molecule, forming a $\mathrm{P}-\mathrm{O}-\mathrm{C}$ bond and liberating a water molecule. ${ }^{20,39}$

Summarizing the optical spectroscopy investigation of both nanostructures, the most likely modification of the MPA molecule during wire or ribbon formation is the substitution of $\mathrm{H}$ atoms in $\mathrm{OH}$ groups of this molecule, with possible interactions with $\mathrm{Al}$ atoms and/or incorporation of ethyl radicals from the ethanol. In other words, the XPS, IR, and Raman analysis of both nanowires and nanoribbons substantiate the hypothesis that they are essentially formed by MPA, modified by the incorporation of $\mathrm{Al}$ atoms and ethyl radicals into its structure. Within such picture, aluminum diethyl methyl phosphonate would be the proposed material forming both nanowires and nanoribbons.

\section{CONCLUSION}

In a broad manner, the discovery of a new material can always be seen from two different perspectives: a technologic and a scientific one. From the technological pointof-view, the importance of such material is rated by its potential applications arising from its physical properties (optical, electrical, morphological, and etc...). From the scientific approach though, the assessment of such properties themselves justifies the investigation. Therefore, every report on a new material tries to trail both, or at least one, of these perspectives. Nevertheless, it is the eventual forthcoming interest of the scientific community which will dictate the importance of such material. Returning to the first paragraph of this paper, the story behind the discovery of carbon nanotubes is emblematic: multi-walled carbon nanotubes were initially described as early as 40 years prior to the work of Iijima. ${ }^{2,40}$ However, only in the early $90 \mathrm{~s}$, during the dawning of the 'nano' age with its scientific and technological interests, carbon nanotubes were 're-discovered' and are, since then, one of the most important materials of the 'nano' world. In other words, any new material is only as important as its consideration by its contemporary scientific community.

Considering the above paragraph and that this paper reports on some physical properties of novel nanostructures formed by methylphosphonic acid. Also considering the large amount of information about them gathered from morphological, structural, and spectroscopical techniques, including a suggestion for their composition, then this work could be positively analyzed from both technological and scientific perspectives. However, it is difficult, and even useless, trying to evaluate their importance for upcoming nanoscience and nanotechnology. Several questions still remain unanswered, or their answer still needs further confirmation, such as the atomic structure of a nanoribbon and a nanowire or their precise composition, respectively. Nevertheless, even more general questions were raised during this work, namely the development necessity of techniques like X-ray diffraction and NMR towards structure determination of solid-state materials in nanogram quantities. Therefore, in a pragmatic view, one should restrain himself/herself to all the specific information and discussion about nanoribbons and nanowires presented in this work, which surely constitute a characteristic contribution to both nanoscience and nanotechnology fields.

Acknowledgments: The authors acknowledge financial support from CNPq, Fapemig, LNLS and Instituto de Nanociências/MCT. B. S. A., L. A. S. C., M. R., F. A. C. O., D. M. R., and L. G. C. are thankful to CNPq and Capes for their scholarship.

\section{References and Notes}

1. H. W. Kroto, J. R. Heath, S. C. Obrien, R. F. Curl, and R. E. Smalley, Nature 318, 162 (1985).

2. S. Iijima, Nature 354, 56 (1991).

3. J. A. Schwarz, C. I. Contescu, and K. Putyera (eds.), Dekker Encyclopedia of Nanoscience and Nanotechnology, Marcel Dekker, NewYork (2004).

4. R. M. Black, R. J. Clarke, R. W. Read, and M. T. J. Reid, J. Chromatography A 662, 301 (1994).

5. G. W. Wagner, P. W. Bartram, O. Koper, and K. J. Klabunde, J. Phys. Chem. B 103, 3225 (1999).

6. T. A. Lewington, M. R. Alexander, G. E. Thompson, and E. McAlpine, Surface Engineering 18, 228 (2002).

7. W. Gao, L. Dickinson, C. Grozinger, F. G. Morin, and L. Reven, Langmuir 12, 6429 (1996).

8. G. N. Fontes and B. R. A. Neves, Langmuir 21, 11113 (2005).

9. G. Cao, H.-G. Hong, and T. E. Mallouk, Acc. Chem. Res. 25, 420 (1992). 
10. K. Maeda, Y. Kiyozumi, and F. Mizukami, Angew. Chem. Int. Ed. Engl. 33, 2335 (1994).

11. K. Maeda, J. Akimoto, Y. Kiyozumi, and F. Mizukami, Angew. Chem. Int. Ed. Engl. 34, 1199 (1995).

12. K. Maeda, Y. Kiyozumi, and F. Mizukami, J. Phys. Chem. B 101, 4402 (1997).

13. K. Maeda, L. McCusker, and Ch. Baerlocher, Stud. Surf. Sci. Catal. 135, 290 (2001).

14. R. Zeng, X. Fu, C. Gong, and Y. Sui, J. Mater. Sci. 41, 4771 (2006).

15. I. V. Romanova, I. A. Farbun, V. S. Aleksandrova, and S. A. Khainakov, Rus. J. Appl. Chem. 76, 731 (2003).

16. S. S. Bao, G. S. Chen, Y. Wang, Y. Z. Li, L. M. Zheng, and Q. H. Luo, Inorg. Chem. 45, 1124 (2006).

17. D. E. Lansky, P. Y. Zavalij, and S. R. J. Oliver, Acta Cryst. C 57, 1051 (2001).

18. G. Philippin, J. Delhalle, and Z. Mekhalif, Appl. Surf. Sci. 212, 530 (2003).

19. K. Popov, H. Ronkkomaki, and L. H. J. Lajunen, Pure Appl. Chem. 73, 1641 (2001).

20. S. N. Magonov and M.-H. Whangbo, Surface Analysis with STM and AFM, VCH Publishers, New York (1996).

21. P. Atkins, Physical Chemistry, W. H. Freeman and Company, NewYork (1994).

22. G. H. B. Thompson, Physics of Semiconducting Lasers, Wiley, NewYork (1980).

23. H.-R. Chen, J.-L. Shi, Y. Yang, Y.-S. Li, D.-S. Yan, and C.-S. Chi, Appl. Phys. Lett. 81, 2761 (2002).

24. D. Cummins, G. Boschloo, M. Ryan, D. Corr, R. S. Nagaraja, and D. Fitzmaurice, J. Phys. Chem. B 104, 11449 (2000).
25. N. W. Ashcroft, Solid State Physics, Saunders College, Philadelphia (1976).

26. D. R. Lide (ed.), Handbook of Chemistry and Physics, 75th edn., CRC Press, Boca Raton (1990).

27. B. E. Warren, X-ray Diffraction, Dover Publications, NewYork (1969).

28. B. D. Cullity, Elements of X-ray Diffraction, Addison-Wesley, NewYork (1978).

29. J. M. Hollas, Modern Spectroscopy, John Wiley and Sons, Chichester (1992).

30. P. R. Davies and N. G. Newton, Appl. Surf. Sci. 181, 296 (2001).

31. A. Viinikanoja, J. Lukkari, T. Aaritalo, T. Laiho, and J. Kankare, Langmuir 19, 2768 (2003).

32. J. H. Scofield, J. Electron Spectrosc. 8, 129 (1976).

33. G. Socrates, Infrared Characteristic Group Frequencies, Wiley, Chichester (1994).

34. R. D. Ramsier, P. N. Henriksen, and A. N. Gent, Surf. Sci. 203, 72 (1988).

35. F. Berger, E. Brunol, R. Planade, and A. Chambaudet, Thin Solid Films 436, 1 (2003).

36. J. M. Bowen, C. R. Powers, A. E. Ratliffe, M. G. Rockley, and A. W. Hounslow, Environ. Sci. Technol. 22, 1178 (1988).

37. B. Aurian-Blajeni and M. M. Boucher, Langmuir 5, 170 (1989).

38. M. K. Templeton and W. H. Weinberg, J. Amer. Chem. Soc. 107, 97 (1985).

39. The Raman spectra of EMP and DEMP, including their structure, are available at the Sigma Aldrich website (www.sigmaaldrich.com).

40. M. Monthioux and V. L. Kuznetsov, Carbon 44, 1621 (2006).

Received: 20 December 2006. Revised/Accepted: 21 February 2007. 